
\title{
25 Research Soure \\ Multi-Omics Analysis Identifies the Heterogeneity and Prognosis in Bladder Cancer
}

\section{Wenchao Ma}

Shanghai Tenth People's Hospital

\section{Cheng Li}

Shanghai Tenth People's Hospital

\section{Yuchao Liu}

Shanghai Tenth People's Hospital

\section{Wentao Zhang}

Shanghai Tenth People's Hospital

\section{Yadong Guo}

Shanghai Tenth People's Hospital

\section{Fuhan Yang}

Shanghai Tenth People's Hospital

Junfeng Zhang

Shanghai Tenth People's Hospital

\section{Hong Wang}

Shanghai Tenth People's Hospital

\section{Weihua Jia}

Anhui Medical University

\section{Xudong Yao}

Shanghai Tenth People's Hospital

\section{Jun Xiao}

The First Affiliated Hospital of USTC: Anhui Provincial Hospital

\section{Yang Yan ( 2133213@tongji.edu.cn )}

Shanghai Tenth People's Hospital https://orcid.org/0000-0001-7484-0211

\section{Primary research}

Keywords: DNA methylation, multi-omics, bladder cancer, single nucleotide variants, copy number variations

Posted Date: November 2nd, 2021

DOI: https://doi.org/10.21203/rs.3.rs-1023596/v1 
License: (c) (i) This work is licensed under a Creative Commons Attribution 4.0 International License. Read Full License 


\section{Abstract \\ Background}

The multi-omics integrated analysis can help researchers understand the biological behavior of bladder cancer( $\mathrm{BCa})$ in a more systematic and comprehensive manner, and further provide new clues for finding valuable tumor markers and therapeutic targets.

\section{Methods}

In this study, we applied the DNA methylation data to construct a prognosis classifier and stratified the BCa patients into high- and low-risk subtype. The differences of transcriptome, single nucleotide variants and copy number variations between two subgroups were explored for finding the changes of molecular mechanism.

\section{Results}

With 18 pairs DNA methylation samples, ten differentially methylated positions(DMPs) were identified and applied to evaluate the risk score of each sample. Kaplan-Meier survival analysis displayed that BCa patients with high risk had a poor prognosis than the lower $(\mathrm{p}<0.0001)$. In transcriptome analysis, many immune related pathways and biological process changed between high- and low-risk patients. The results also displayed that naive $B$ cells, plasma cells, $C D 8+T$ cells and $T$ cell regulatory(Tregs) infiltrated less in high-risk patients and these patients were less sensitive to immunotherapy and chemotherapy. As for single nucleotide variants, we found that TP53, CDKN1A, STAG2 and other genes were more frequently mutated in high-risk BCa patients. Only copy number variation in high-risk patients were displayed for the limitation of TCGA data.

\section{Conclusions}

The high- and low-risk patients identified by DNA methylation data of bladder cancer were significant different in survival. The comprehensive comparison of multi-omics data between subgroups can help clinicians find the heterogeneity of tumor biological behavior and contribute to precision treatment in bladder cancer.

\section{Introduction}

Bladder cancer( $\mathrm{BCa}$ ) which is a superficial, easy to relapse and high mortality malignant tumor derives from bladder mucosa(1). The incidence of bladder cancer ranks No.2 in genitourinary tumors(2). According to Global Cancer Statistics 2020, new BCa patients accounted for 3\%(573,278 cases) of all malignant tumors, and 212,536 patients died of bladder cancer in 2020(3). In clinical, BCa is divided into 
non-muscular invasive bladder cancer(NMIBC) and muscular invasive bladder cancer(MIBC)(4). Although comprehensive therapy was applied for the treatment of NMIBC, $10-30 \%$ patients would progress to MIBC in five years, which is more malignant(5). Therefore, it is very urgent to explore the molecular mechanism of bladder cancer.

In the past, the bioinformation analysis of bladder cancer limited to single omics, and it can't reflect the molecular characteristics completely(6). Multi-omics integrated analysis can help researchers greater understand the features of disease, from the original reasons to the functional consequences and therapy options $(7,8)$. It is reported that the methylation level of the whole genome of tumor cells is closely related to disease progression and malignancy(9). Chen $X$ et al. has successfully developed the urine DNA methylation examination method which is applied for early diagnosis and recurrence monitor of bladder cancer(10). Previous studies also indicated that immune cell infiltration in tumors was depended on tumor cell-intrinsic factors and involved in the response to immunotherapy $(11,12)$. In addition, the single nucleotide variants(SNVs) and copy number variations(CNVs) in genome level were also crucial for the occurrence of malignant cancers(13). For example, the single nucleotide variants which reflected the variation of tumor genome drive tumor occurrence and impact the prognosis and therapeutic effect(14). Compared with any other type of somatic genetic changes, copy number variation has a greater impact on the cancer genome(15).

Finally, we selected the DNA methylation data to evaluate the risk score of BCa patients, stratified the patients into two subtypes and explored the differences of transcriptome, single nucleotide variants and copy number variations between two subgroups.

\section{Methods}

\section{Data selection and pre-processing}

For this study, DNA methylation data(including $415 \mathrm{BCa}$ samples and 21 corresponding normal adjacent samples) and 408 corresponding clinical information were downloaded from The Cancer Genome Atlas (TCGA) GDC data portal. RNA-sequencing counts, simple nucleotide variants(SNV) and copy number variation(CNV) of BCa patients were also retrieved from the TCGA GDC data portal. The DNA methylated samples missing data over $80 \%$ and the methylated positions missing data over $50 \%$ were excluded. After removing outlier samples, 18 pairs BCa methylation data were selected for further differential analysis. For methylated BCa data, only $345 \mathrm{BCa}$ methylated samples were retained after removing repeated samples and normalized samples containing NA.

\section{Establishment of risk assessment model and survival analysis}

The R package "CHAMP"(16) was applied to normalize the DNA methylated data and perform the difference analysis. Univariate and multivariate cox regression analysis were applied to filter differentially methylated positions closely related to the survival of BCa patients. The risk score was generated by 
"predict" function in R. The Kaplan-Meier survival analysis was used to estimate the survival difference between high and low risk groups.

\section{Transcriptome data analysis}

After filtering, 341 patients(169 high risk and 172 low risk) were extracted from the TCGA database for transcriptome data. The KEGG and GO Enrichment Analysis were performed by R package "clusterProfiler". The CIBERSORT algorithm was a deconvolution algorithm based on gene expression and was used to quantify the relative scores of 22 tumor infiltrating immune cells. CIBERSORT P value was derived for the deconvolution of each sample using Monte Carlo sampling and samples with $\mathrm{P}<0.05$ were selected for further analysis. In order to calculate the correlation between gene expression and DNA methylation positions, we mapped the average beta value of the probe to the promoter region and gene body, including TSS200 (region from -200 bp upstream to the transcription start site (TSS) itself), 1stExon (the first exon), TSS1500 (from -200 to -1500 bp upstream of TSS) and 5'UTR. The subclass mapping and Tumor Immune Dysfunction and Exclusion (TIDE) algorithm were applied to evaluate the effect of immune checkpoint inhibitors. The Genomics of Drug Sensitivity in Cancer(GDSC) and R package "pRRophetic" were used to evaluate the chemotherapeutic response BCa patients. According to the PRISM Repurposing dataset and the Cancer Therapeutics Response Portal(CTRP2.0) drug sensitivity AUC value data and the expression profile data in Broad Institute-Cancer Cell Line Encyclopedia(CCLE) project, the potential therapeutic drugs were predicted for TCGA subgroups.

\section{Single Nucleotide Variants and Copy Number Variation}

The R package "maftools"(17) was used to describe the single nucleotide variant profiles of the BCa patients. In addition, we calculated the tumor mutation burden(TMB) of each sample through dividing the total number of variations by total exon length. The copy number variation of the BCa patients was visualized by "RCircos" R package(18).

\section{Results}

\section{Identification DMPs and Construction DNA Methylation Prognosis Classifier}

In TCGA BCa data, there are 21 pairs DNA methylated samples(21 BCa samples and 21 corresponding normal adjacent samples) in total. After removing the outlier samples, 18 pairs DNA methylation samples were selected for further analysis(Figure 2A). Firstly, we applied the R package "CHAMP" to normalize the DNA methylated data and perform the differential analysis. With the criteria of deltaBeta $>0.45$ and $p<0.001,2285$ differentially methylated positions(DMP) were identified(Figure 2B, Supplementary Table S1). Among 415 BCa DNA methylated samples, 4 duplicated, 5 low-quality and 61 NA-containing samples were discarded(Figure 1). Therefore, 345 DNA methylated samples were retained for downstream analysis. The univariate Cox proportional hazard regression model was applied to analyze 2285 differentially methylated positions. As a result, $50 \mathrm{DMPs}(\mathrm{p}<0.05)$ were taken into multivariate Cox regression analysis(Supplementary Table S2). Finally, 10 DMPs were applied to construct the DNA 
methylation prognosis classifier (Supplementary Table S3). Kaplan-Meier survival analysis displayed that BCa patients with high risk had a poor prognosis $(p<0.0001$, Figure $2 C)$.

\section{KEGG Pathway and GO Term Function Enrichment Analysis}

In 345 BCa samples, 341 corresponding RNA sequencing data(169 high risk and 172 low risk) were retrieved from the TCGA database. The differential KEGG pathways between high and low risk groups were neuroactive ligand receptor interaction, chemical carcinogenesis receptor activation, melanoma, thyroid hormone synthesis, primary immunodeficiency, complement and coagulation cascades, tryptophan metabolism and carbohydrate digestion and absorption(Figure 3A). The top three differential cellular components(CC) were external side of plasma membrane, blood microparticle and lamellar body(Figure 3B). Humoral immune response, antimicrobial humoral response and negative regulation of response to wounding were the top three differential biological process(Figure 3C). The differential molecular functions(MF) between groups were shown in Figure 3D.

\section{Evaluation of Tumor-infiltrating Immune Cells}

In order to understand the immune microenvironment of BCa tissues, we evaluated 22 tumor infiltrating immune cells of 341 tumor samples by CIBERSORT. With the CIBERSORT $P<0.05$, the proportion of 22 tumor infiltrating immune cells of 173 patients(88 high risk and 85 low risk) were displayed in Figure 4A. In order to further determine the differences between different groups, we compared 22 tumor infiltrating immune cells between high-risk and low-risk patients(Figure 4B). The results showed that naive B cells, plasma cells, CD8+ T cells and T cell regulatory(Tregs) infiltrated more in low-risk patients.

\section{The Correlation Between DNA Methylation Positions and Host Gene Expression}

For the purpose of identifying the correlation between DNA methylation position and host gene expression, we calculated the Pearson correlation coefficient of ten DNA methylation positions and corresponding host gene expression. The results showed that the methylation of cg06551997 and cg07568841 negatively correlated with the expression of SLFN12L and ZNRF2, respectively(Figure 5A, $5 B)$. On the contrary, the expression of COL23A1, NR2E1 and TFAP2B were positively correlated with the methylation level of cg08560734, cg06433023 and cg22282405(Figure 5C, 5C, 5D), separately.

\section{Differences in Sensitivity to Immune/Chemotherapy Between High- and Low-risk BCa}

Previous studies have confirmed that CTLA-4, PD-1 and PD-L1 inhibitors show significant anti-tumor responses in the treatment of advanced bladder cancer(19). To identify the sensitivity difference to immune checkpoint inhibitors between high and low risk BCa patients, the TIDE algorithm was applied to evaluate the response of 341 patients to immunotherapy. The results showed that low-risk patients $(37.21 \%, 64 / 172)$ may be more likely to respond to immunotherapy than high-risk patients $(23.08 \%$, $39 / 169)(P=0.004753$, Figure $6 A)$. Besides, subclass mapping was also applied to evaluate the sensitivity of two BCa subtypes to immunotherapy(CTLA-4 and PD-1). As shown in Figure 6B, PD-1 inhibitors may be more effective for low-risk subtype. 
As the important method for BCa therapy, chemotherapy plays a key role in improving the prognosis of patients with BCa. Therefore, we applied the cell line data in GDSC and the transcriptome data in TCGA to predict the IC50 of each sample through ridge regression model. The results indicated that low risk patients with $\mathrm{BCa}$ were more sensitive to many chemotherapeutic drugs including methotrexate(Figure 7).

For the precision treatment of $\mathrm{BCa}$, we applied CTRP and PRISM- derived drug response data to identify drug candidates with higher drug sensitivity in patients with high or low risk scores. Firstly, we performed differential drug response analysis between the groups with high-risk scores (top decile) and low risk scores (bottom decile) to identify compounds in the high risk group with lower estimated AUC values or compounds in the low risk group with lower estimated AUC values $(\log 2 \mathrm{FC}>0.10)$. And then, potential compounds were selected by evaluating the spearman correlation coefficient between the AUC value and the risk score (Spearman's $r<-0.2$ or $>0.2$ for CTRP and PRISM). The results displayed that high-risk patients were more sensitive to poziotinib. However, mitoxantrone and PI-103 may be more effective for patients with low risk scores(Supplementary Figure 1).

\section{Differences in Single Nucleotide Variants and Copy Number Variation Between High- and Low-risk Bladder Cancer}

The mutation is involved in the occurrence and progression of tumors, and affect the prognosis of patients. To explore the SNV, we separately described the single nucleotide variant profiles of the high-

and low-risk patients(Figure 8). As shown in Figure 9A, the top ten mutation genes were TP53(15\%), $\operatorname{TTN}(14 \%), \operatorname{KDM6A}(10 \%), \operatorname{KMT} 2 \mathrm{D}(9 \%), \operatorname{MUC16}(9 \%), \operatorname{PIK} 3 \mathrm{CA}(9 \%), \operatorname{ARID1A}(7 \%), \operatorname{RB} 1(7 \%), \operatorname{MACF} 1(6 \%)$ and CDKN1A(5\%) in high risk patients. In low risk patients(Figure 9B), the top ten mutation genes were TTN(15\%), TP53(11\%), KMT2D(10\%), MUC16(9\%), SYNE1(9\%), FGFR3(8\%), RB1(7\%), KDM6A(7\%), FAT4 $(6 \%)$ and ARID1A(5\%). In addition, we found there was no statistical difference in TMB between high-risk and low-risk BCa(Supplementary Figure 2). As for the copy number variation, variation occurs on most chromosomes except for chromosomes 10, 15 and $Y$ in high-risk BCa patients(Figure 10).

\section{Discussion}

In recent years, high-throughput sequencing technology and bioinformatics have developed rapidly, and clinicians have gradually realized that many diseases were difficult to describe in a single theoretical model(20). Therefore, researchers have tried to apply multi-omics integrated analysis to reveal the biological processes and molecular mechanisms of diseases, thereby confirming the drug target of the disease and providing a basis for individualized treatment and medication guidance(21, 22). The TCGA database collected the genome, epigenome and transcriptome information from over 30 cancer types(23), which provides the possibility for researchers to conduct multi-omics integrated analysis of tumors. In this study, we applied the DNA methylation data of bladder cancer for risk stratification and compared the differences of transcriptome, single nucleotide variants and copy number variation between high- and low-risk patients. Through multi-omics comparison, we aim to reveal molecular 
differences between high- and low-risk patients and make a contribution to the drug development and precise treatment.

As we all know, DNA methylation is an extensively studied epigenetic modification, which changes the activity of DNA fragments without changing the sequence by adding methyl to DNA molecules(24). In general, DNA methylation is regulated by methylases and demethylases and the main function is to regulate gene expression(25). Specifically. the higher the methylation level of $\mathrm{CpG}$ islands in the promoter region of the gene, the expression level of the corresponding gene is relatively lower. In recent years, a large number of studies have shown that abnormal DNA methylation is closely related to the occurrence and progression of tumors(26). In malignant tumors, the DNA methylation changes mainly manifest that the methylation of tumor suppressor genes and DNA repair genes cause tumor suppressor gene silencing and repair genes inactivation, so that the inhibitory effect on tumors is lost and gene damage increases; while the methylation of overall genome decrease activates the proto-oncogene and the retrotransposon and decreases the chromosome stability(27). Therefore, we selected the DNA methylation data to construct the prognosis classifier and stratified the patients with risk score.

According to the 18 pairs DNA methylation data and corresponding clinical information, we identified ten DMPs closely related to the survival of BCa patients and constructed the DNA methylation prognosis classifier. The result of the Kaplan-Meier survival analysis displayed high-risk patients had a poor prognosis than the lower. In transcriptome analysis, many immune related pathways and biological process and tumor infiltrating immune cells changed between high- and low-risk patients. Therefore, we speculate that the immune-related changes may be part of the reason for the poor prognosis of high-risk patients. For example, previous studies have demonstrated that the changes of tryptophan metabolism can promote the mobility of cancer cells and weaken the anti-tumor response of the immune system(28$30)$. Researchers have also proved that $B$ cells can activate the $T$ cells and produce antibodies which inhibit the tumors(31-33). In this study, we also found there were fewer tumor-infiltrating naive B cells, plasma cells, CD8+ T cells and regulatory $T$ cells(Tregs) in high-risk patients when compared with the lower. Previous studies have demonstrated that these immune cells were involved in the composition of tumor microenvironment and regulated the progression of the tumors. For instance, B cells were associated with survival and involved in the immunotherapy response of melanoma(34), renal cell carcinoma(35) and sarcoma(36). The CD8+ T cells were critical executor in the anti-tumor effects of immune cells(37). However, the differentiation and function of CD8+ T cells infiltrated in tumors were often disturbed $(38,39)$. Immune checkpoint inhibitor, such as programmed death receptor 1(PD-1) which can block the co-inhibitory receptors on the surface of depleted CD8+ T cells, can reactivate the cytolysis of $T$ cells and exert anti-tumor effects(40). The cytotoxic $T$ lymphocyte-associated antigen-4(CTLA-4) inhibitor can selectively eliminate regulatory $T$ cells in the tumor microenvironment to inhibit tumor progression(41). At the same time, the predictive results of immunotherapy also demonstrated that lowrisk patients were more sensitive to immune checkpoint inhibitors.

The somatic mutation and copy number variation were important sources of the genomic variation(42, 43). Comparison of tumor genome variations in different subgroups can reveal tumor heterogeneity and 
provide direction for precise tumor treatment. In this study, we found that TP53, MACF1, CDKN1A, EP300, STAG2, FAT3, MKI67 and ANK3 were more frequently mutated in high-risk BCa patients. The mutated TP53 can promote tumorigenesis because it cannot prevent cell division with DNA damage and chromosomal aberrations(44). The CDKN1A gene, which is involved in the regulation of the cell cycle, is regulated by $\mathrm{p} 53$ in response to various stress stimuli and exists mutations in bladder cancer(45). As for the CNV, we only explored the copy number variation in high-risk bladder cancer for the limitation of TCGA data.

The purpose of this research is to stratify the BCa patients and reveal the molecular features of each subtype through multi-omics integrated analysis. However, this research still exists some shortcomings. Firstly, only DNA methylation, RNA sequencing, SNVs and CNVs data of BCa patients were analyzed in this study. The TCGA database does not include the proteome, metabolome and microbiome data. Secondly, the copy number variation was only analyzed in high-risk patients for the limitation of TCGA data. Finally, no external verification was performed in this study. In the future, we hope to incorporate more comprehensive omics data for joint analysis and perform external validation.

\section{Conclusion}

We applied the DNA methylation data of bladder cancer to construct a prognosis classifier and divided patients into high- and low-risk subtypes. The transcriptome data analysis revealed the differences of pathways, GO functions, tumor-infiltrating immune cells between two subtypes. The analysis of drug sensitivity demonstrated that high-risk patients were resistance to immunotherapy/chemotherapy. The TP53 and CDKN1A mutation were more frequently in high-risk patients.

\section{Abbreviations}

BCa: bladder cancer, DMP: differentially methylated positions, NMIBC: non-muscular invasive bladder cancer, MIBC: muscular invasive bladder cancer, TCGA: the Cancer Genome Atlas, SNV: simple nucleotide variants, CNV: copy number variation, TSS: transcription start site, TIDE: Tumor Immune Dysfunction and Exclusion, GDSC: Genomics of Drug Sensitivity in Cancer, CTRP: Cancer Therapeutics Response Portal, CCLE: Cancer Cell Line Encyclopedia, TMB: tumor mutation burden, CC: cellular component, MF: molecular function, PD1: programmed death receptor 1, CTLA-4: cytotoxic T lymphocyte-associated antigen-4,

\section{Declarations}

\section{Acknowledgements}

Not applicable.

\section{Authors' contributions}


Data curation, WM, YL, FY, Formal analysis, WM, CL, WZ, Methodology, WM, CL, JZ, WJ, Software, WM, HW, Writing, WM, YG, Writing-review and editing, WM, XY, JX, YY. All authors contributed to the article and approved the manuscript.

\section{Funding}

Clinical Special Project of Shanghai Municipal Health Commission (202040179), Shanghai Science Committee Foundation (19411967700), National Natural Science Foundation of China Youth Project, project approval number: 81802554 .

\section{Availability of data and materials}

All samples were downloaded from the TCGA database(https ://cancergenome.nih.gov/).

\section{Ethics approval and consent to participate}

Not applicable.

\section{Consent for publication}

Not applicable.

\section{Competing interests}

All authors have declared no conflict of interests.

\section{Author details}

${ }^{1}$ Department of Urology, Shanghai Tenth People's Hospital, Tongji University, Shanghai, 200072, China

${ }^{2}$ Shanghai Clinical College, Anhui Medical University, Hefei, China

${ }^{3}$ Institute of Urinary Oncology, School of Medicine, Tongji University, Shanghai, China

${ }^{4}$ Department of Urology, The First Affiliated Hospital of USTC, Division of Life Sciences and Medicine, University of Science and Technology of China, Hefei, 230001, China

${ }^{5}$ School of Life Science, Anhui Medical University, Hefei, Anhui, 230032, China

\section{References}

1. Lenis A, Lec P, Chamie K, Mshs MJJ. Bladder Cancer: A Review. (2020) 324:1980-91. doi: 10.1001/jama.2020.17598

2. Antoni S, Ferlay J, Soerjomataram I, Znaor A, Jemal A, Bray FJEu. Bladder Cancer Incidence and Mortality: A Global Overview and Recent Trends. (2017) 71:96-108. doi: 
10.1016/j.eururo.2016.06.010

3. Sung H, Ferlay J, Siegel R, Laversanne M, Soerjomataram I, Jemal A, et al. Global Cancer Statistics 2020: GLOBOCAN Estimates of Incidence and Mortality Worldwide for 36 Cancers in 185 Countries. (2021) 71:209-49. doi: 10.3322/caac. 21660

4. Cumberbatch $M$, Jubber I, Black P, Esperto F, Figueroa J, Kamat A, et al. Epidemiology of Bladder Cancer: A Systematic Review and Contemporary Update of Risk Factors in 2018. (2018) 74:784-95. doi: 10.1016/j.eururo.2018.09.001

5. Zheng Z, Mao S, Zhang W, Liu J, Li C, Wang R, et al. Dysregulation of the Immune Microenvironment Contributes to Malignant Progression and Has Prognostic Value in Bladder Cancer. (2020) 10:542492. doi: 10.3389/fonc. 2020.542492

6. Hasin Y, Seldin M, Lusis AJGb. Multi-omics approaches to disease. (2017) 18:83. doi: 10.1186/s13059-017-1215-1

7. Civelek M, Lusis AJNrG. Systems genetics approaches to understand complex traits. (2014) 15:3448. doi: $10.1038 / \mathrm{nrg} 3575$

8. Sun Y, Hu YJAig. Integrative Analysis of Multi-omics Data for Discovery and Functional Studies of Complex Human Diseases. (2016) 93:147-90. doi: 10.1016/bs.adgen.2015.11.004

9. Ehrlich MJE. DNA hypomethylation in cancer cells. (2009) 1:239-59. doi: 10.2217/epi.09.33

10. Chen X, Zhang J, Ruan W, Huang M, Wang C, Wang H, et al. Urine DNA methylation assay enables early detection and recurrence monitoring for bladder cancer. (2020) 130:6278-89. doi: 10.1172/jci139597

11. WES Analysis Measures Immune Infiltration and Predicts Therapy Response. \%J Cancer discovery. (2021). doi: 10.1158/2159-8290.Cd-rw2021-131

12. Li J, Byrne K, Yan F, Yamazoe T, Chen Z, Baslan T, et al. Tumor Cell-Intrinsic Factors Underlie Heterogeneity of Immune Cell Infiltration and Response to Immunotherapy. (2018) 49:178-93.e7. doi: 10.1016/j.immuni.2018.06.006

13. Schepeler T, Lamy P, Hvidberg V, Laurberg J, Fristrup N, Reinert T, et al. A high resolution genomic portrait of bladder cancer: correlation between genomic aberrations and the DNA damage response. (2013) 32:3577-86. doi: 10.1038/onc.2012.381

14. Cannataro V, Gaffney S, Townsend JJJotNCl. Effect Sizes of Somatic Mutations in Cancer. (2018) 110:1171-7. doi: 10.1093/jnci/djy168

15. Zack T, Schumacher S, Carter S, Cherniack A, Saksena G, Tabak B, et al. Pan-cancer patterns of somatic copy number alteration. (2013) 45:1134-40. doi: 10.1038/ng.2760

16. Butcher L, Beck SJM. Probe Lasso: a novel method to rope in differentially methylated regions with 450K DNA methylation data. (2015) 72:21-8. doi: 10.1016/j.ymeth.2014.10.036

17. Mayakonda A, Lin D, Assenov Y, Plass C, Koeffler HJGr. Maftools: efficient and comprehensive analysis of somatic variants in cancer. (2018) 28:1747-56. doi: 10.1101/gr.239244.118 
18. Zhang H, Meltzer P, Davis SJBb. RCircos: an R package for Circos 2D track plots. (2013) 14:244. doi: 10.1186/1471-2105-14-244

19. Cheng W, Fu D, Xu F, Zhang ZJO. Unwrapping the genomic characteristics of urothelial bladder cancer and successes with immune checkpoint blockade therapy. (2018) 7:2. doi: 10.1038/s41389017-0013-7

20. Reuter J, Spacek D, Snyder MJMc. High-throughput sequencing technologies. (2015) 58:586-97. doi: 10.1016/j.molcel.2015.05.004

21. Lindskrog S, Prip F, Lamy P, Taber A, Groeneveld C, Birkenkamp-Demtröder K, et al. An integrated multi-omics analysis identifies prognostic molecular subtypes of non-muscle-invasive bladder cancer. (2021) 12:2301. doi: 10.1038/s41467-021-22465-w

22. Cantini L, Zakeri P, Hernandez C, Naldi A, Thieffry D, Remy E, et al. Benchmarking joint multi-omics dimensionality reduction approaches for the study of cancer. (2021) 12:124. doi: 10.1038/s41467020-20430-7

23. JN. Comprehensive genomic characterization defines human glioblastoma genes and core pathways. (2008) 455:1061-8. doi: 10.1038/nature07385

24. Greenberg M, Bourc'his DJNrMcb. The diverse roles of DNA methylation in mammalian development and disease. (2019) 20:590-607. doi: 10.1038/s41580-019-0159-6

25. Smith Z, Meissner AJNrG. DNA methylation: roles in mammalian development. (2013) 14:204-20. doi: $10.1038 / \mathrm{nrg} 3354$

26. Schübeler DJN. Function and information content of DNA methylation. (2015) 517:321-6. doi: $10.1038 /$ nature14192

27. Koch A, Joosten S, Feng Z, de Ruijter T, Draht M, Melotte V, et al. Analysis of DNA methylation in cancer: location revisited. (2018) 15:459-66. doi: 10.1038/s41571-018-0004-4

28. Sadik A, Somarribas Patterson L, Öztürk S, Mohapatra S, Panitz V, Secker P, et al. IL 411 Is a Metabolic Immune Checkpoint that Activates the AHR and Promotes Tumor Progression. (2020) 182:125270.e34. doi: 10.1016/j.cell.2020.07.038

29. Liu X, Shin N, Koblish H, Yang G, Wang Q, Wang K, et al. Selective inhibition of IDO1 effectively regulates mediators of antitumor immunity. (2010) 115:3520-30. doi: 10.1182/blood-2009-09246124

30. Tang K, Wu Y, Song Y, Yu BJJoh, oncology. Indoleamine 2,3-dioxygenase 1 (ID01) inhibitors in clinical trials for cancer immunotherapy. (2021) 14:68. doi: 10.1186/s13045-021-01080-8

31. Conejo-Garcia J, Biswas S, Chaurio RJSii. Humoral immune responses: Unsung heroes of the war on cancer. (2020) 49:101419. doi: 10.1016/j.smim.2020.101419

32. Chen V, Greenberger B, Taylor J, Edelman M, Lu BJljoro, biology, physics. The Underappreciated Role of the Humoral Immune System and B Cells in Tumorigenesis and Cancer Therapeutics: A Review. (2020) 108:38-45. doi: 10.1016/j.jijrobp.2020.03.022 
33. Garaud S, Buisseret L, Solinas C, Gu-Trantien C, de Wind A, Van den Eynden G, et al. Tumor infiltrating B-cells signal functional humoral immune responses in breast cancer. (2019) 5. doi:

10.1172/jci.insight.129641

34. Cabrita R, Lauss M, Sanna A, Donia M, Skaarup Larsen M, Mitra S, et al. Tertiary lymphoid structures improve immunotherapy and survival in melanoma. (2020) 577:561-5. doi: 10.1038/s41586-0191914-8

35. Helmink B, Reddy S, Gao J, Zhang S, Basar R, Thakur R, et al. B cells and tertiary lymphoid structures promote immunotherapy response. (2020) 577:549-55. doi: 10.1038/s41586-019-1922-8

36. Petitprez F, de Reyniès A, Keung E, Chen $T$, Sun $C$, Calderaro J, et al. B cells are associated with survival and immunotherapy response in sarcoma. (2020) 577:556-60. doi: 10.1038/s41586-0191906-8

37. Reina-Campos M, Scharping N, Goldrath AJNrl. CD8 T cell metabolism in infection and cancer. (2021). doi: 10.1038/s41577-021-00537-8

38. Philip M, Schietinger AJNrl. CD8 T cell differentiation and dysfunction in cancer. (2021). doi: 10.1038/s41577-021-00574-3

39. Sanmamed M, Nie X, Desai S, Villaroel-Espindola F, Badri T, Zhao D, et al. A Burned-Out CD8 T-cell Subset Expands in the Tumor Microenvironment and Curbs Cancer Immunotherapy. (2021) 11:170015. doi: 10.1158/2159-8290.Cd-20-0962

40. Dolina J, Van Braeckel-Budimir N, Thomas G, Salek-Ardakani SJFii. CD8 T Cell Exhaustion in Cancer. (2021) 12:715234. doi: 10.3389/fimmu.2021.715234

41. Tang F, Du X, Liu M, Zheng P, Liu YJC, bioscience. Anti-CTLA-4 antibodies in cancer immunotherapy: selective depletion of intratumoral regulatory T cells or checkpoint blockade? (2018) 8:30. doi: 10.1186/s13578-018-0229-z

42. Du Toit AJNrM. Copy-number variation. (2020) 18:542. doi: 10.1038/s41579-020-0431-y

43. Ku C, Tan E, Cooper DJJomg. From the periphery to centre stage: de novo single nucleotide variants play a key role in human genetic disease. (2013) 50:203-11. doi: 10.1136/jmedgenet-2013-101519

44. Liu Y, Kwiatkowski DJMct. Combined CDKN1A/TP53 mutation in bladder cancer is a therapeutic target. (2015) 14:174-82. doi: 10.1158/1535-7163.Mct-14-0622-t

45. Cazier J, Rao S, McLean C, Walker A, Walker A, Wright B, et al. Whole-genome sequencing of bladder cancers reveals somatic CDKN1A mutations and clinicopathological associations with mutation burden. (2014) 5:3756. doi: 10.1038/ncomms4756

\section{Figures}


TCGA BCa methylation samples

(21 normal +415 cancer)

(1) remove repeated samples $(n=4)$

(8) remove low-quality samples $(\mathrm{n}=5)$

(3) normalize the methylated data

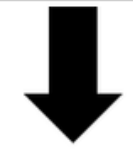

(1) 21 pairs $\mathrm{BCa}$ methylation data

(2) keep survival time $>0(\mathrm{n}=20)$

(4) remove samples containing $\mathrm{NA}(\mathrm{n}=61)$

(3) removing outlier samples $(\mathrm{n}=2)$

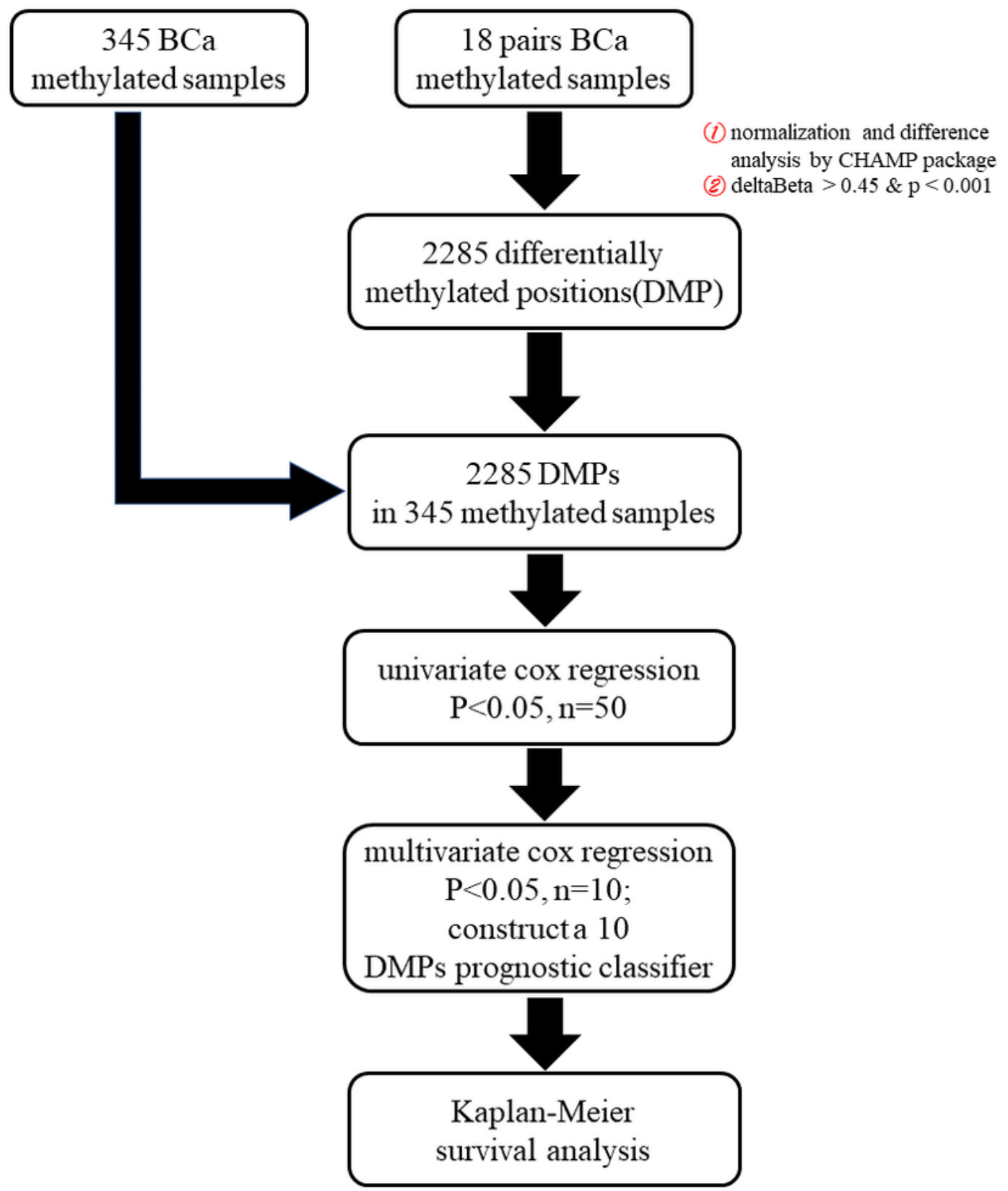

Figure 1

Flow diagram indicating the identification of DMPs and construction DNA methylation prognosis classifier. 

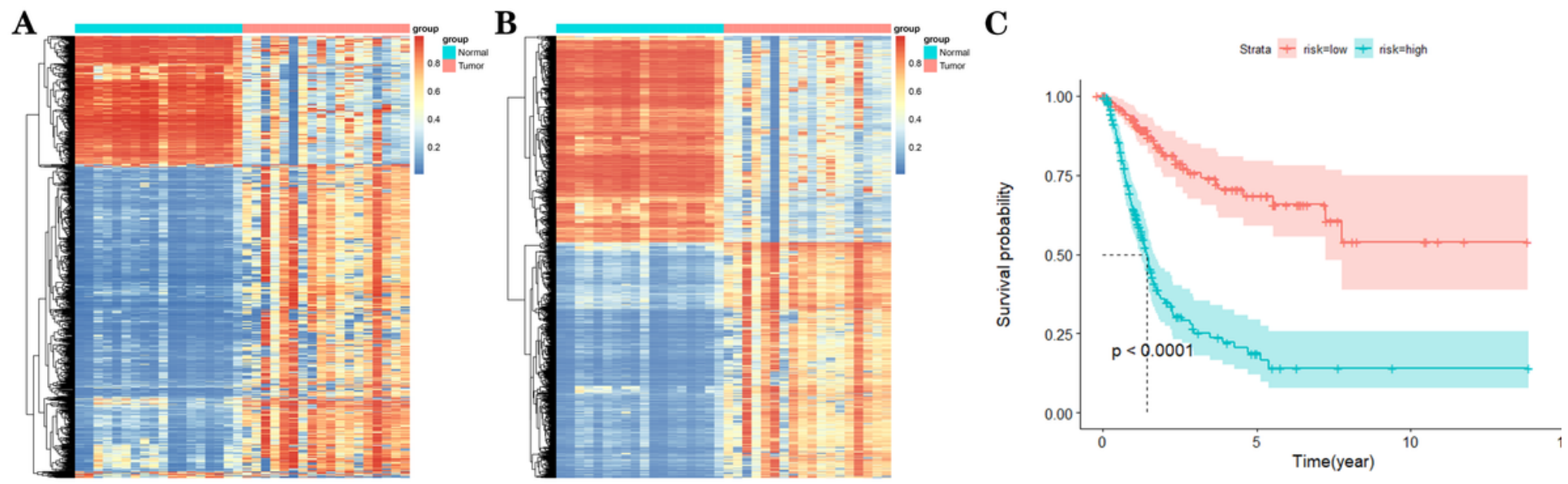

\section{Figure 2}

Identification DMPs and Kaplan-Meier survival based on the classifier. (A) heatmap showing DNA methylation in 18 pairs adjacent-normal and BCa tissues. (B) heatmap showing 2285 differentially methylated positions between 18 pairs adjacent-normal and tumor tissues. (C) Kaplan-Meier survival analysis based on the prognostic classifier.

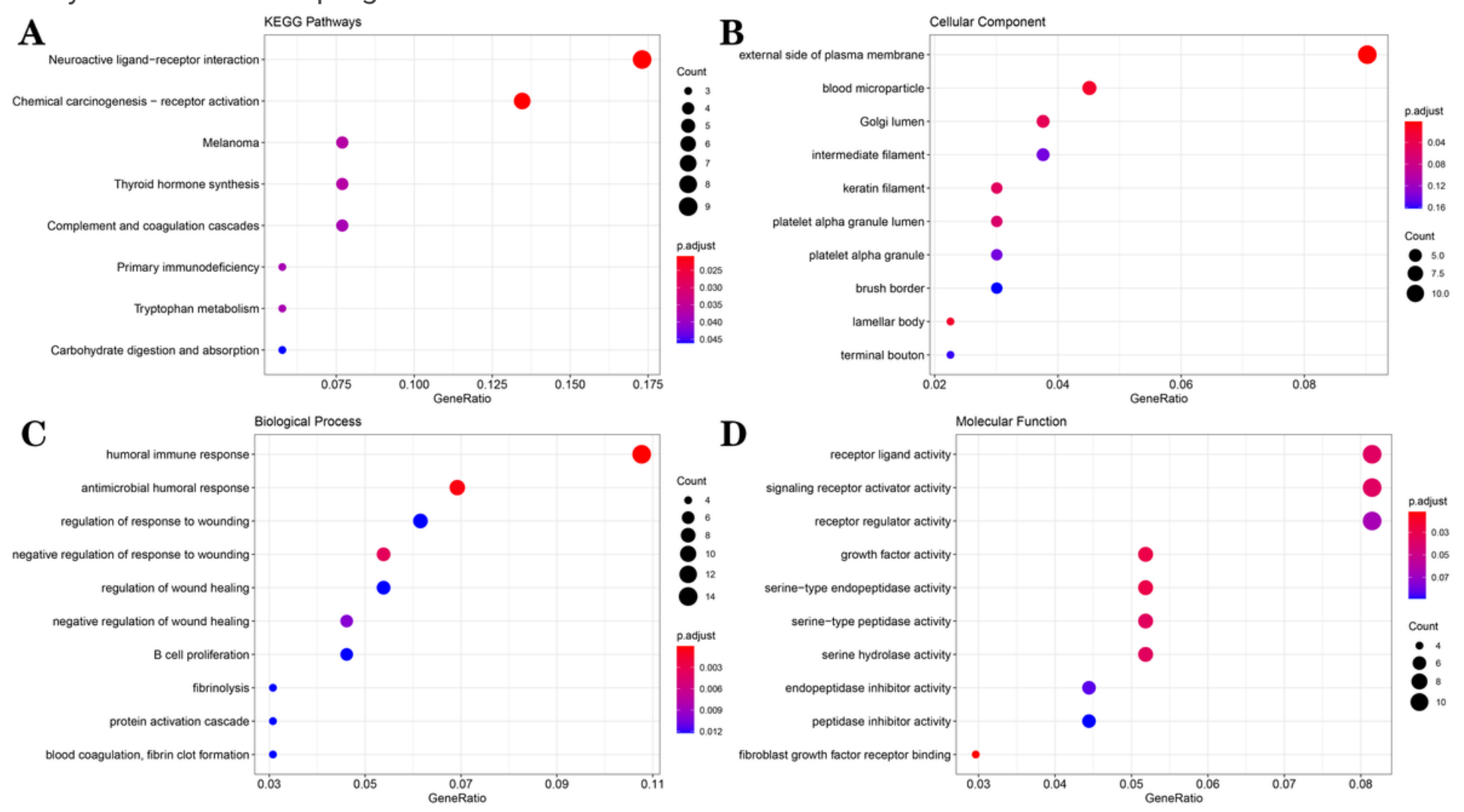

Figure 3

KEGG pathway and GO term enrichment analysis. (A) KEGG pathways. (B) cellular components(CC). (C) biological process(BP). (D) molecular functions(BP). 

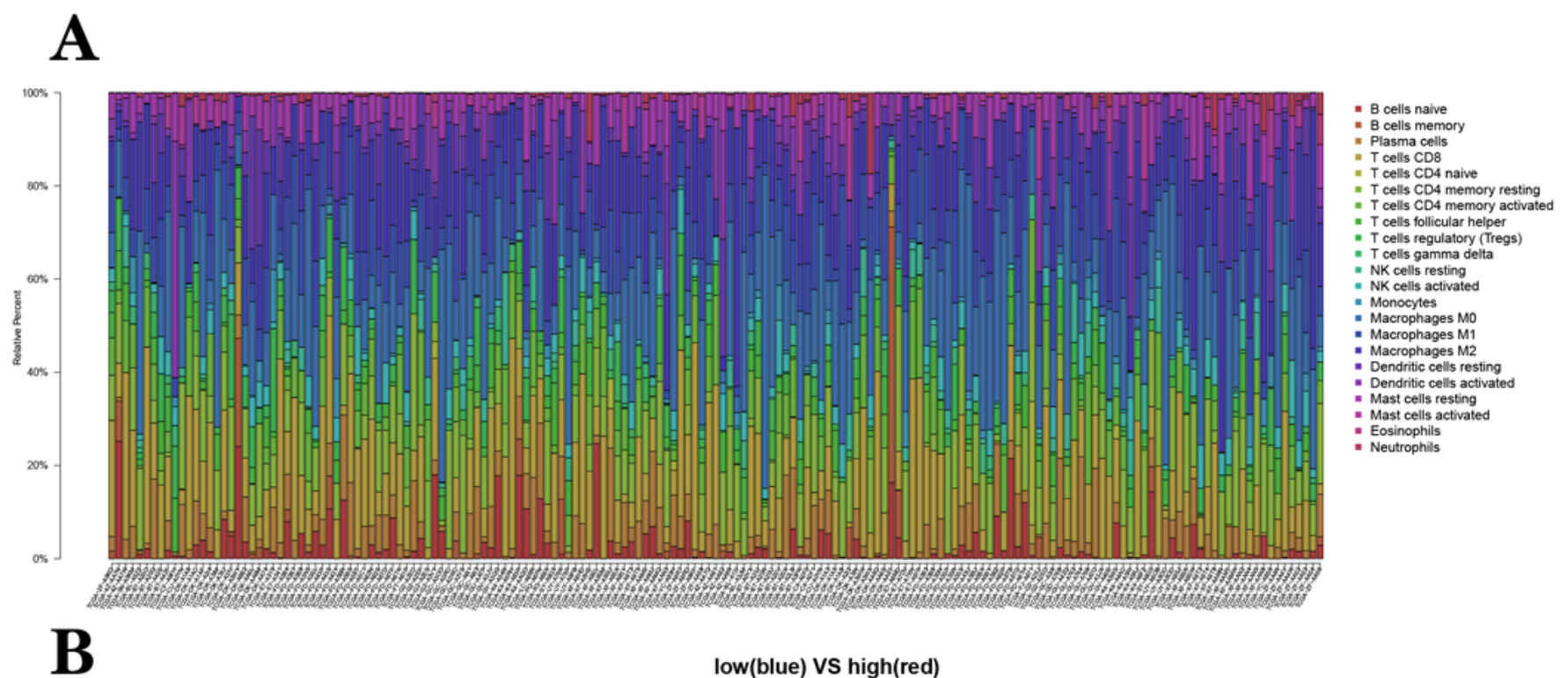

low(blue) Vs high(red)
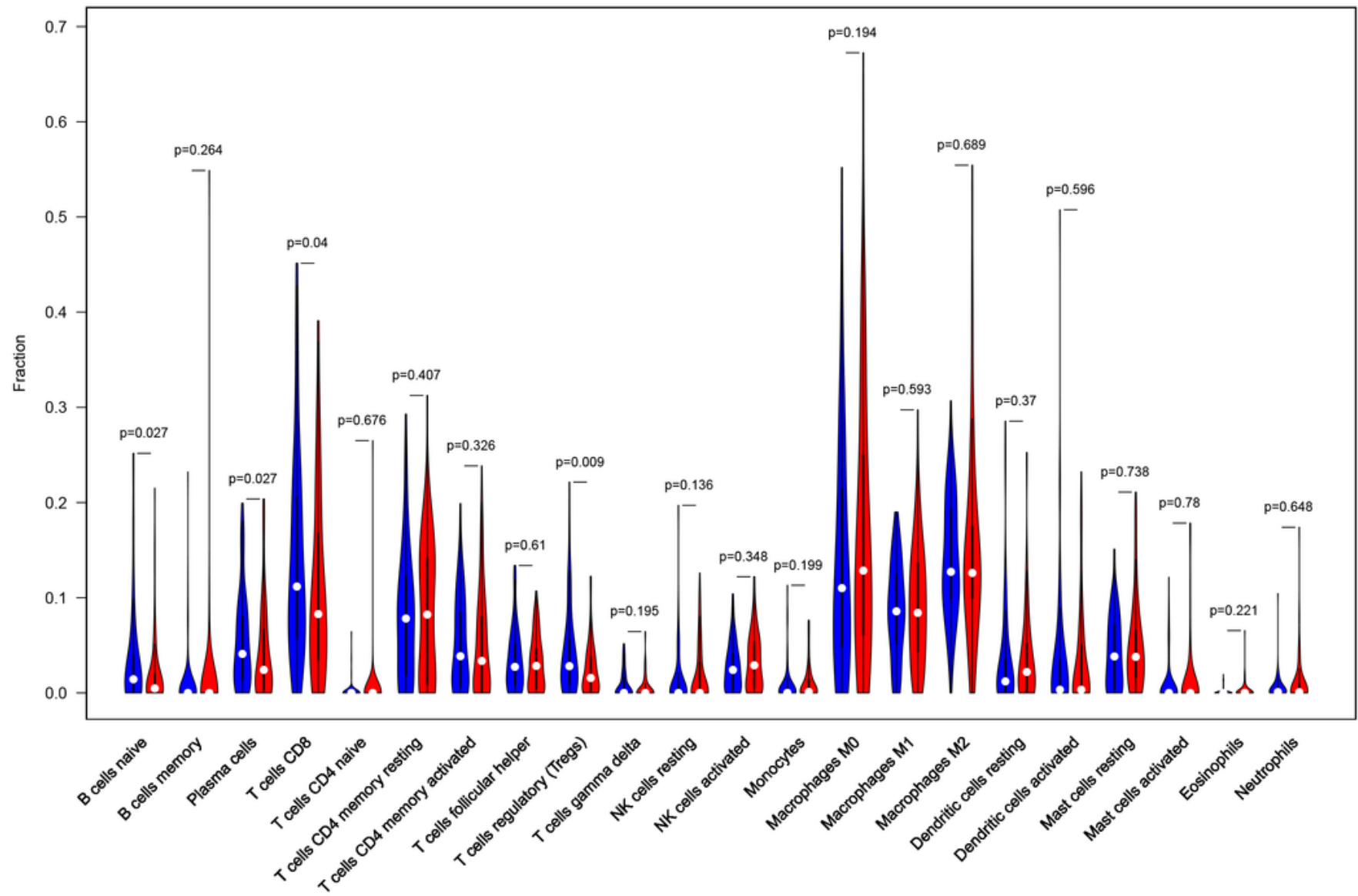

Figure 4

The 22 tumor-infiltrating immune cells in bladder cancer. (A) the proportions of 22 tumor-infiltrating immune cells in 173 samples. (B) the difference of 22 tumor-infiltrating immune cells between high- and low-risk patients. 

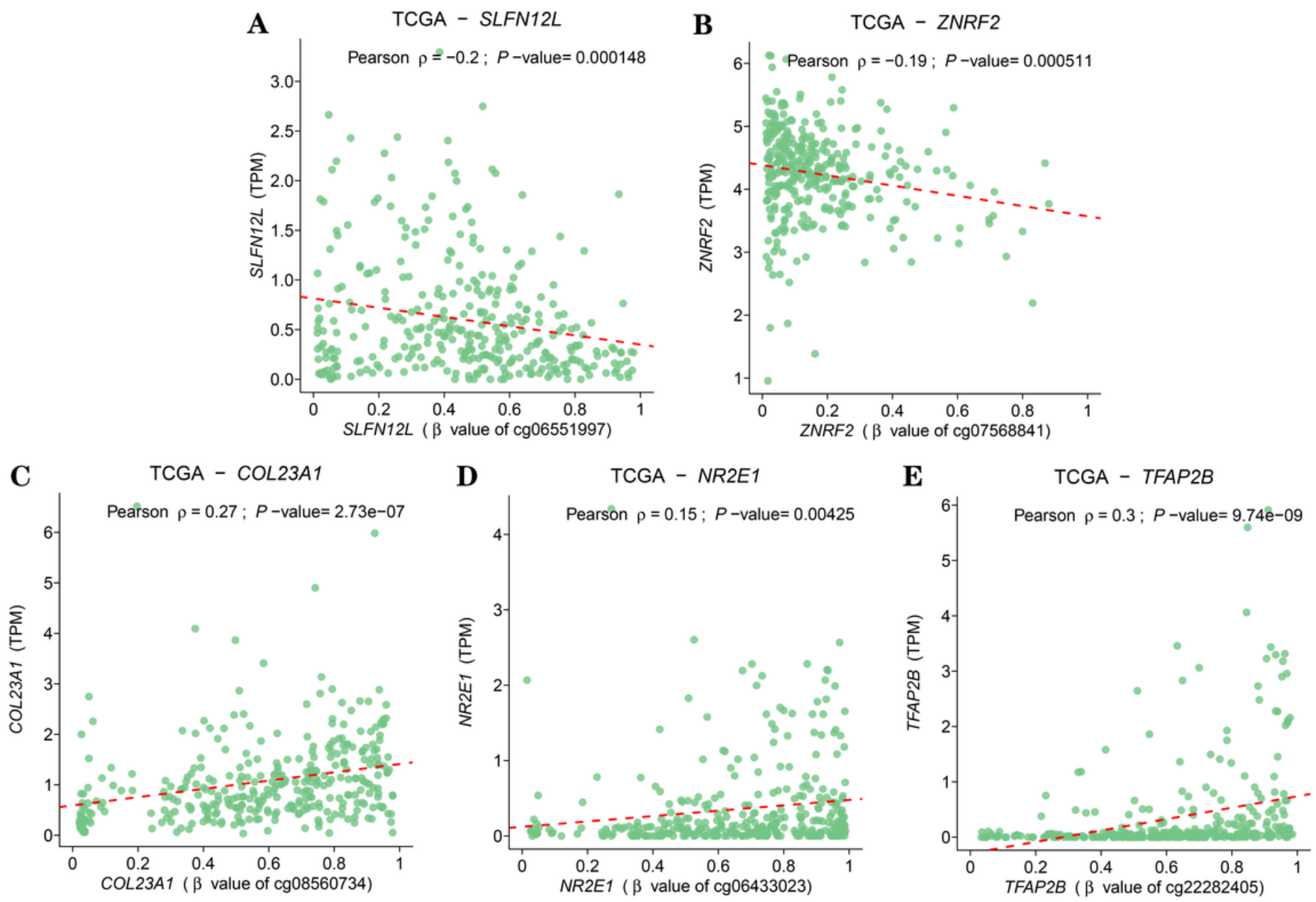

\section{Figure 5}

The correlation between DNA methylation position and host gene expression. (A) cg06551997 and SLFN12L; (B) cg07568841 and ZNRF2; (C) cg08560734 and COL23A1; (D) cg06433023 and NR2E1; (E) cg22282405 and TFAP2B. 
A

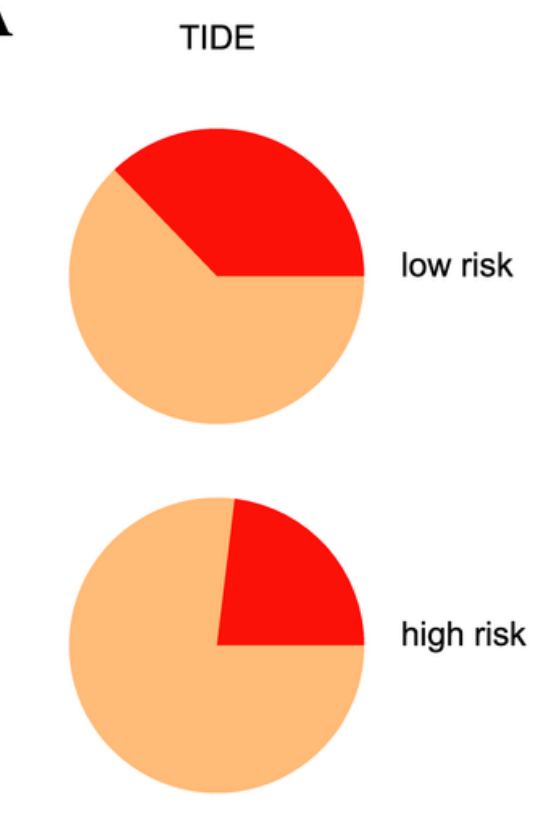

response
B

\section{pvalue}

Nominal $p$ value

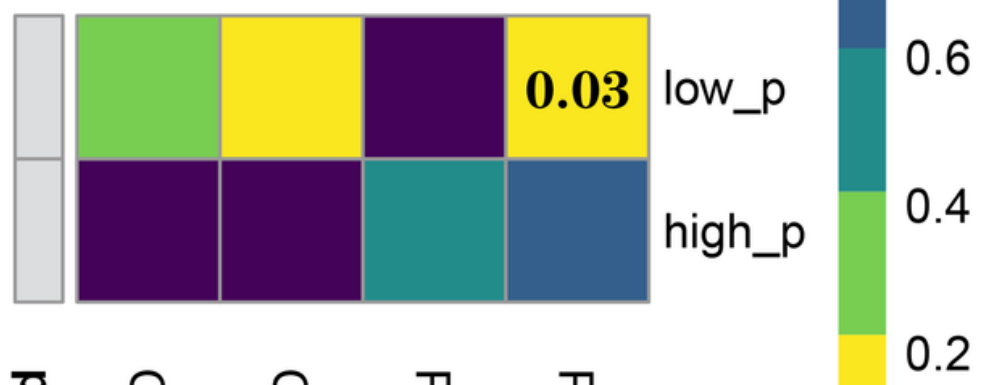

0.2

\section{Figure 6}

The immunotherapeutic sensitivity in high- and low-risk patients. (A) immunotherapeutic response predicted by TIDE algorithm; (B) immunotherapeutic response predicted by subclass mapping in GenePattern. 
A
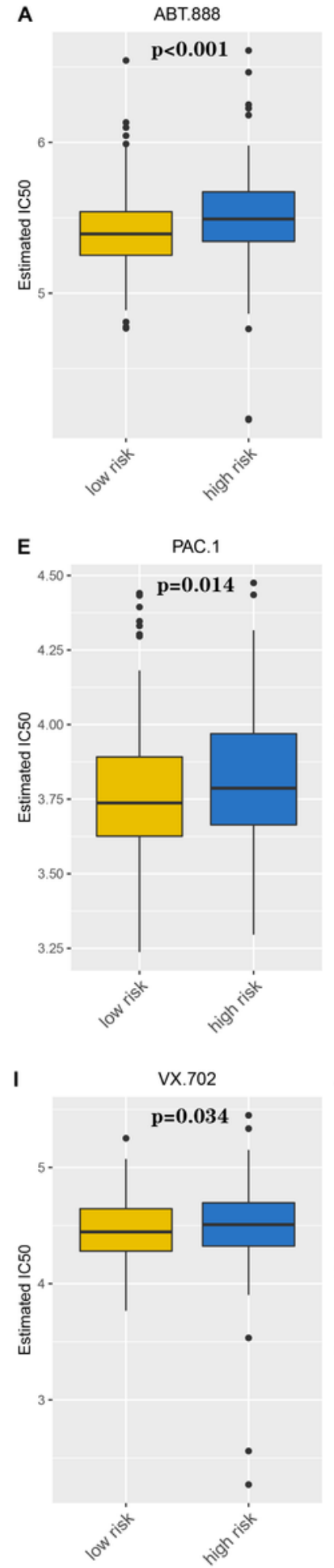
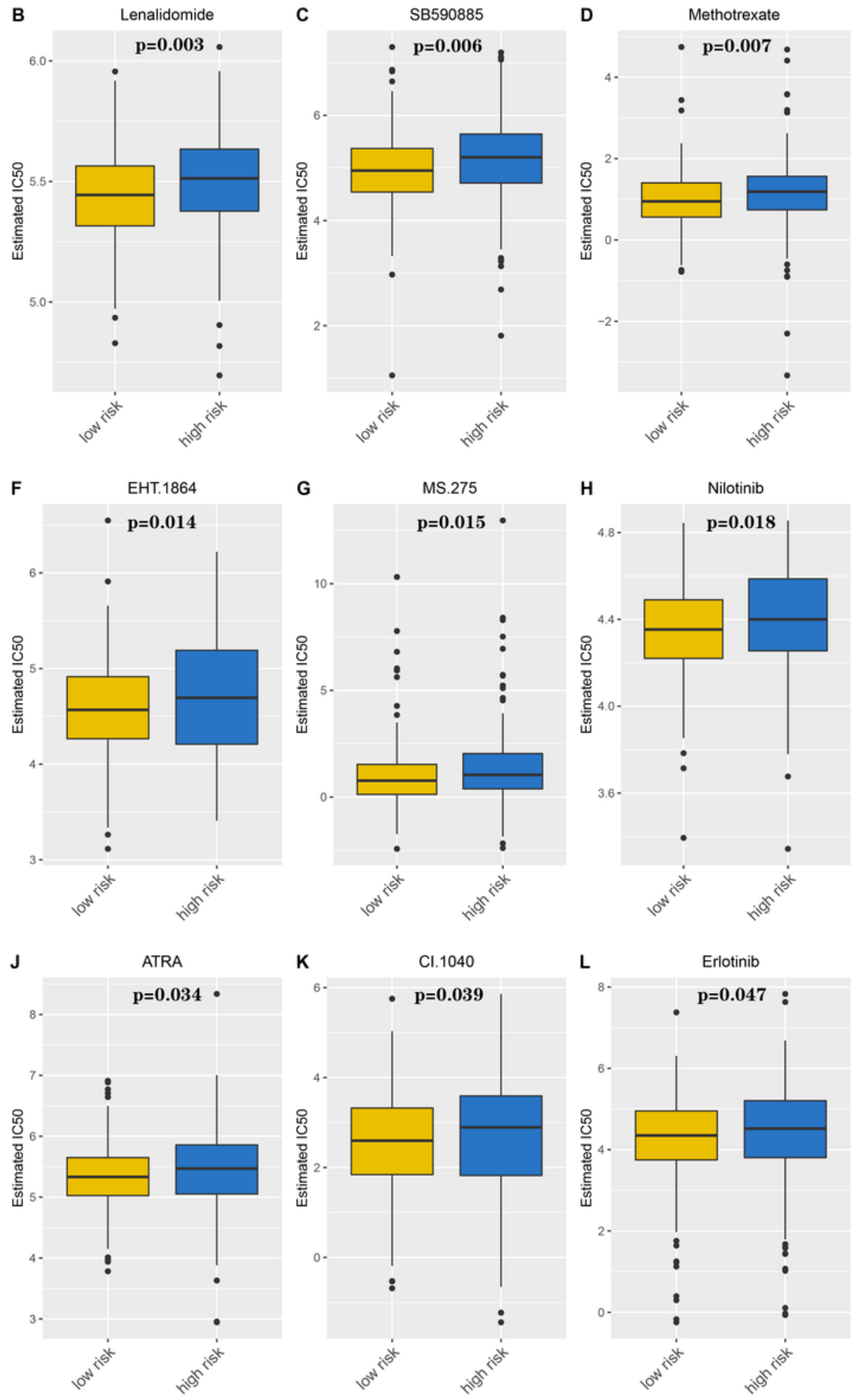

Figure 7

The differences of chemotherapeutic sensitivity between high- and low-risk patients. 
A
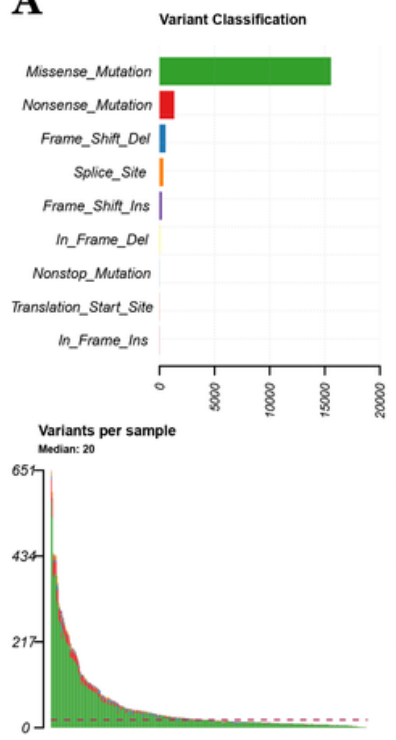
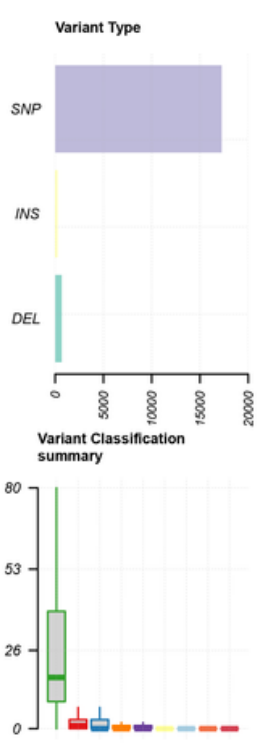

SNV Class

\begin{tabular}{l|l}
$T>G$ & 484 \\
$T>A$ & 794 \\
$T>C$ & 1875
\end{tabular}

$\triangle T \quad 14672$

$C>G \quad 7642$

C>A 2813
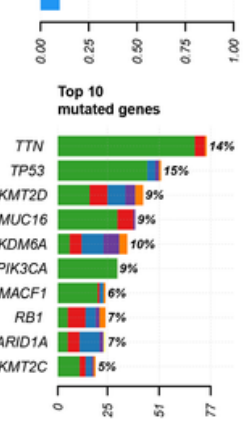

B
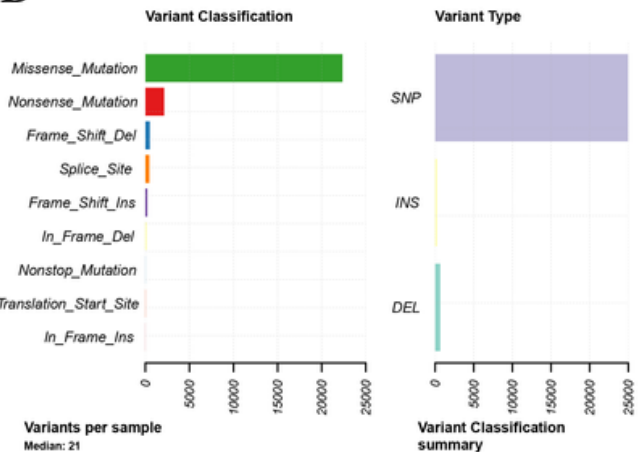

INS
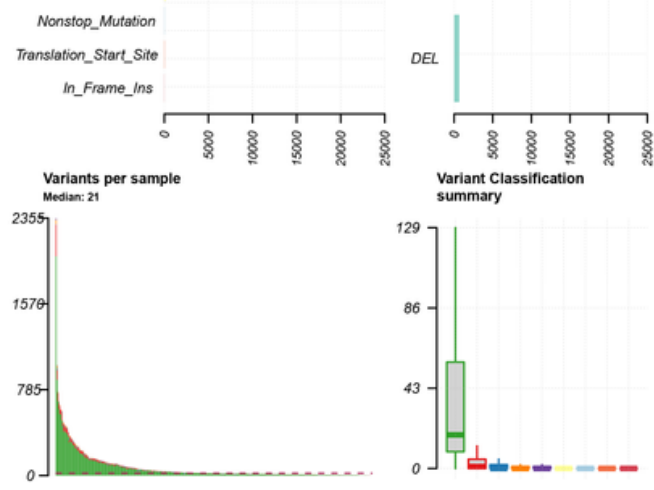

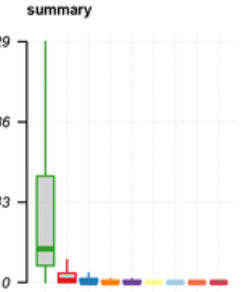

SNV Class

$T>G \mid 1177$

$>A \mid 977$

$T>C \quad 2579$

$\triangle T=20759$

$C>G \quad 9595$

$C>A \quad 4876$

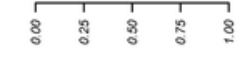

Top 10
mutated genes

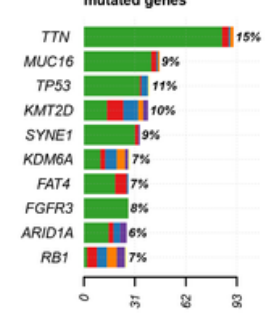

Figure 8

The single nucleotide variants in bladder cancer. (A) the single nucleotide variants in high-risk patients; (b) the single nucleotide variants in low-risk patients. 


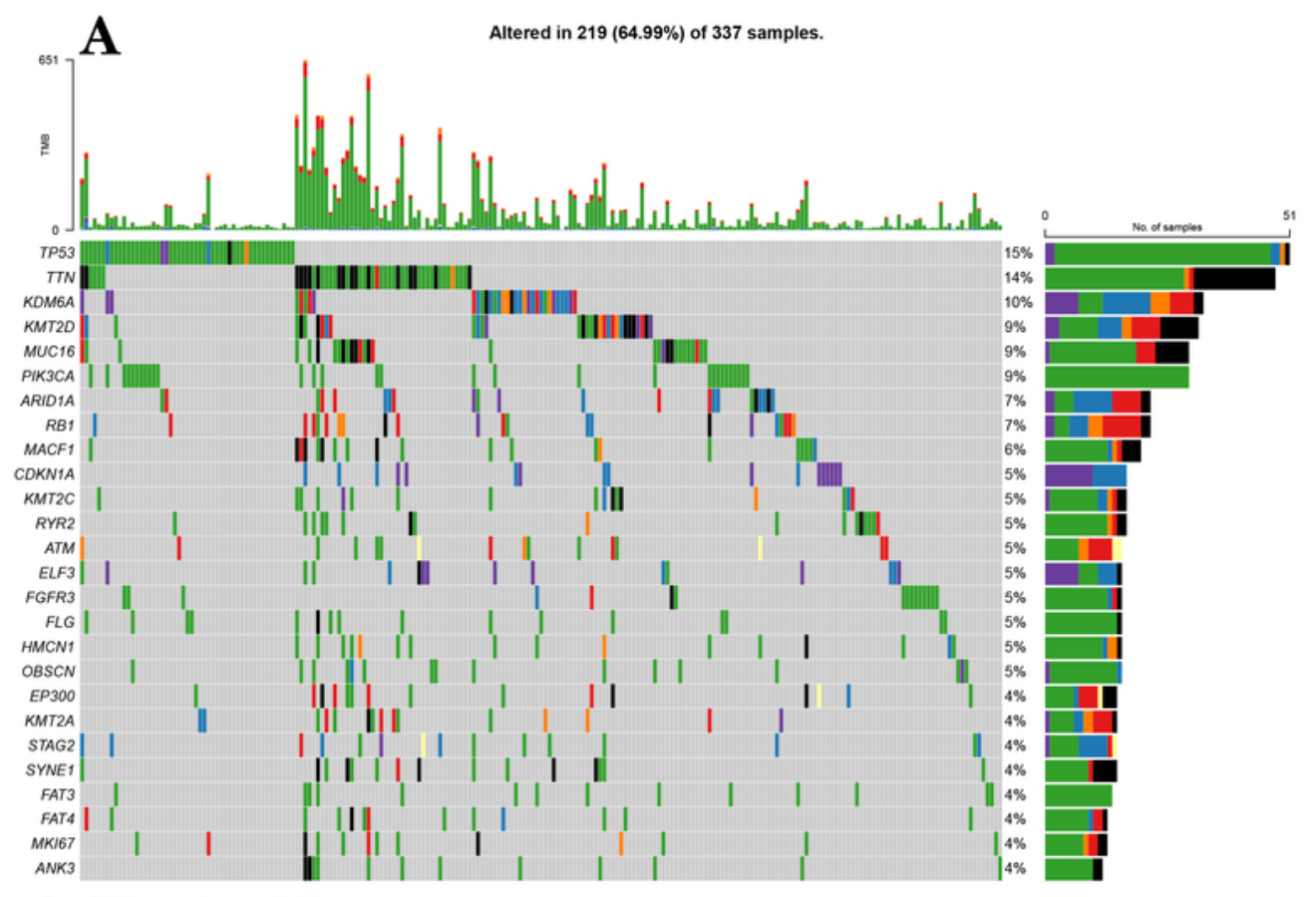

: Frame_Shiting : : Nonsense_Mutation

- Frame_Shit_Del In Frame_Del

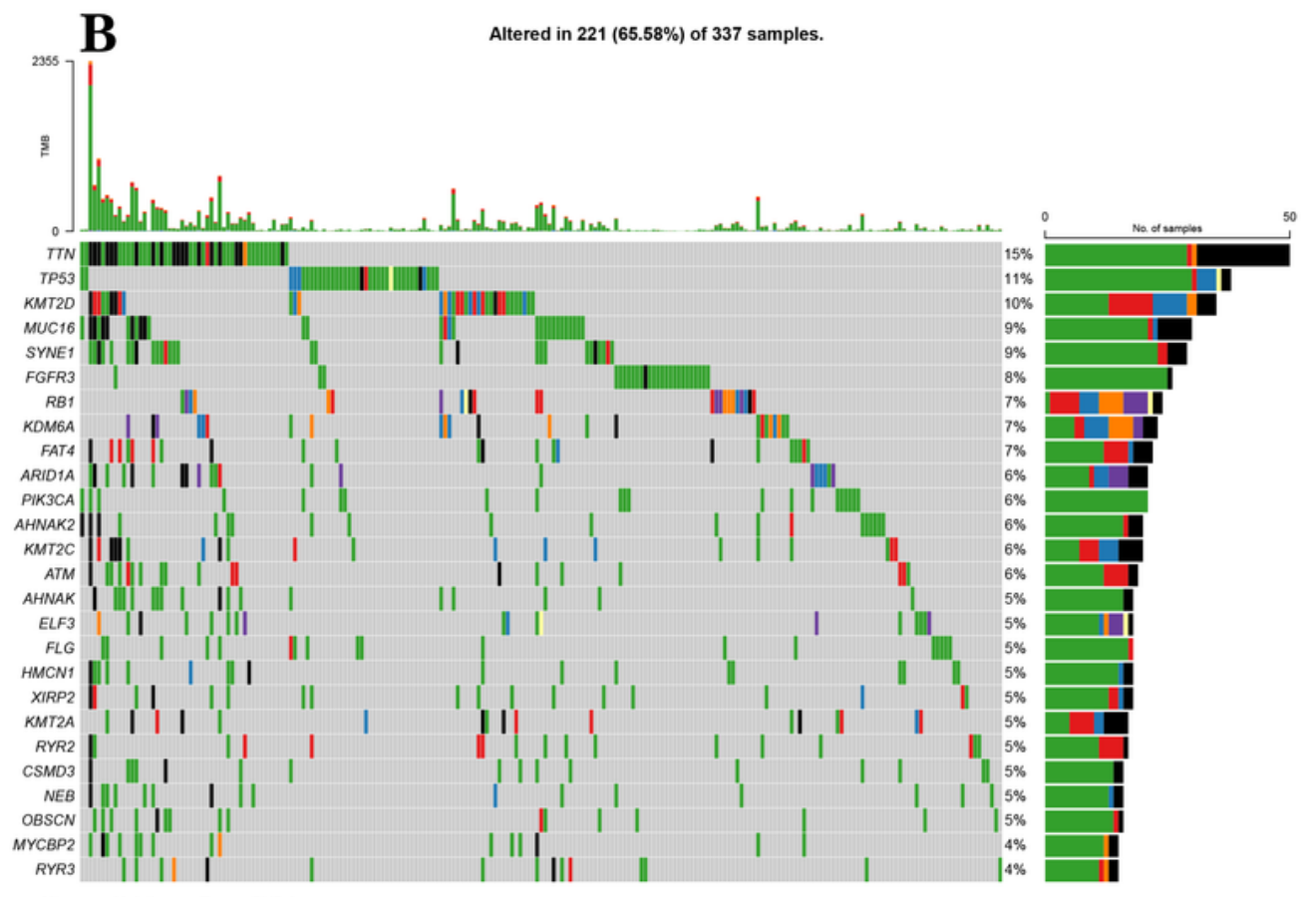

" Missense_Mutation "Frame_ShiftIns

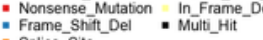

Figure 9

The waterfall charts of single nucleotide variants in bladder cancer. (A) the waterfall chart in high-risk patients; (b) the waterfall chart in low-risk patients. 


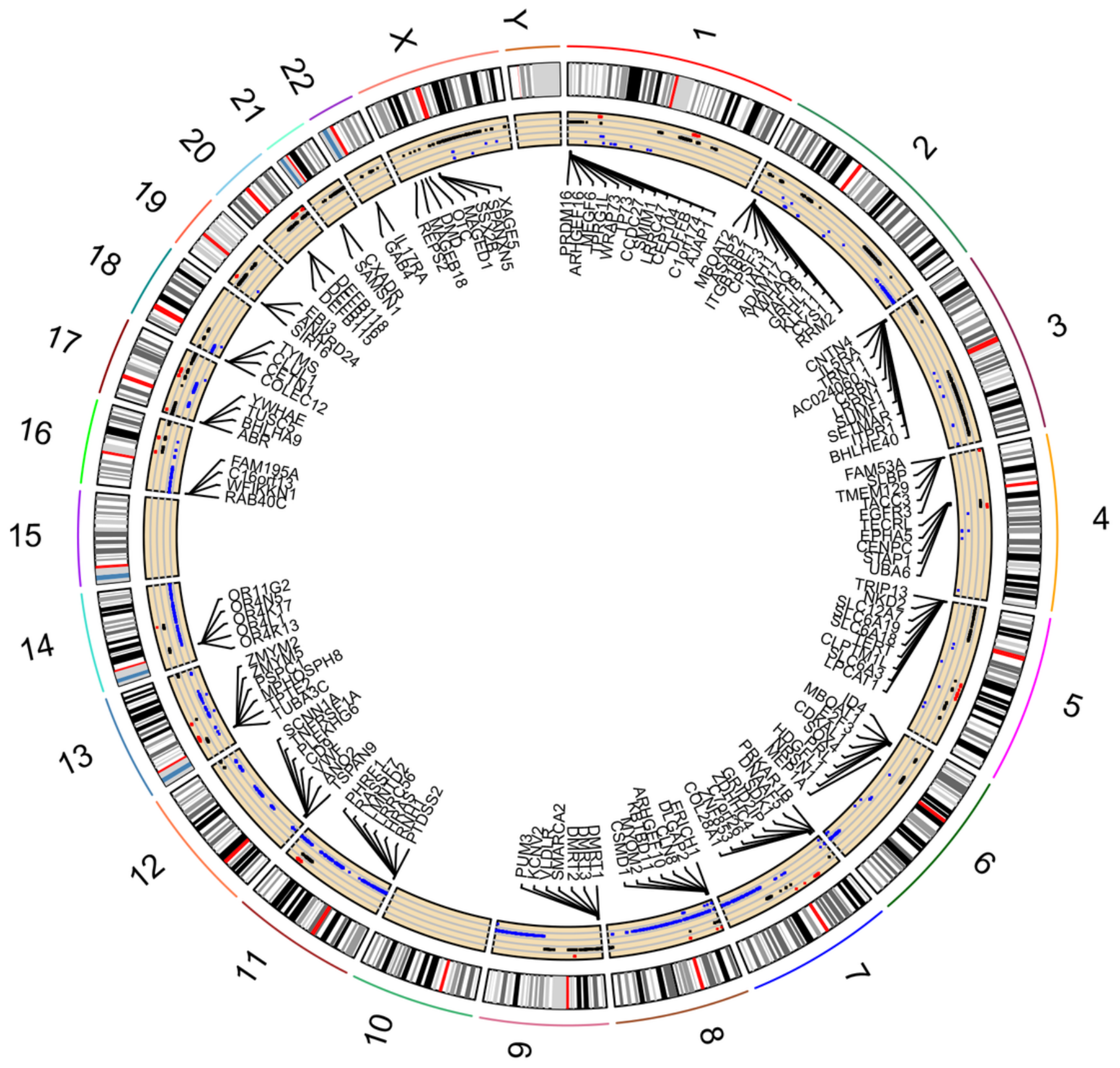

Figure 10

The copy number variations in high-risk patients.

\section{Supplementary Files}

This is a list of supplementary files associated with this preprint. Click to download.

- SupplementaryFigure1.pdf

- SupplementaryFigure2.pdf 
- SupplementaryTable.xlsx

Page 23/23 